\title{
Avaliação do nível de satisfação dos clientes de um restaurante por meio da Teoria de Resposta ao Item
}

Restaurant satisfaction level assessment by Item Response Theory

\author{
Nicásio Gouveia', Fernando De Jesus Moreira Junior", \\ Angela Pellegin Ansuj'", José Renê de Oliveira IV
}

\begin{abstract}
RESUMO
O objetivo desse trabalho foi criar uma escala para avaliar o nível de satisfação dos clientes de um restaurante, por meio do Modelo Logístico de Dois Parâmetros (ML2) da Teoria da Resposta ao Item (TRI). Para isso, foi aplicado um questionário a 640 clientes contendo 28 atributos do restaurante relacionados com os ambientes interno e externo, com a alimentação e com o atendimento. Os resultados mostraram que a maioria dos respondentes estão satisfeitos com os atributos avaliados, sendo que os itens melhores avaliados foram as opções de pagamento, a localização do restaurante e o seu tamanho e estrutura. Observou-se ainda que todos os itens tiveram um bom ajuste com o ML2 da TRI, não sendo necessário a exclusão de nenhum deles. Consequentemente, foi possível construir uma escala interpretável com quatro níveis para a satisfação dos clientes. Além disso, foi possível avaliar o questionário e identificar para qual o intervalo do traço latente que ele é mais adequado. A estimação dos parâmetros permitiu localizar os itens na escala criada, assim como identificar aqueles que merecem maior atenção no planejamento organizacional do restaurante.
\end{abstract}

Palavras-chave: Satisfação do Cliente; Métodos e Modelos Estatísticos; Estatística Aplicada.

\section{ABSTRACT}

The objective of this work was to create a scale to assess the level of customer satisfaction in a restaurant, using the Two Parameter Logistic Model (2PL) of the Item Response Theory (IRT). For this, a questionnaire was applied to 640 customers, including 28 restaurant attributes related to the indoor and outdoor environments, with food and service. The results shown in most respondents are satisfied with the specified effects, with the most recommended items being payment options, a location in the restaurant and its size and structure. Also note that all items had a good fit with the $2 \mathrm{PL}$, so there is no need to exclude them. Consequently, it was possible to build an interpretable scale with four levels for customer satisfaction. In addition, it was possible to evaluate the questionnaire and identify which latent screening interval is most appropriate. The application of permitted adjustments allows to locate the items on the created scale, as well as to identify those that deserve greater attention in the organizational planning of a restaurant.
\end{abstract}

Keywords: Customer Satisfaction; Statistical Methods and Models; Applied Statistics

\footnotetext{
' Universidade Federal de Santa Maria, Santa Maria, Brasil. E-mail: nicasiogouveia@yahoo.com.br.

"Universidade Federal de Santa Maria, Santa Maria, Brasil. E-mail: fmjunior@smail.ufsm.br.

III Universidade Federal de Santa Maria, Santa Maria, Brasil. E-mail: angelaansuj@gmail.com.

Iv Universidade Federal de Santa Maria, Santa Maria, Brasil. E-mail: renerenoli@gmail.com.
} 


\section{INTRODUÇÃO}

A percepção da qualidade baseia-se em critérios objetivos e subjetivos, assim conhecer as expectativa dos clientes pode ser o primeiro passo para o desenvolvimento de ações que levarão a melhorias por parte do gestor da organização (FADEL; REGIS, 2009). O setor de serviços ocupa posição de destaque na economia mundial e para os empreendedores sobreviverem no mercado devem primar pela qualidade dos seus serviços, pois os consumidores estão cada vez mais exigentes e se apóiam em mídias sociais e sites de compartilhamento de avaliações para manifestarem opiniões sobre os serviços experimentados (CORREA; HANSEN, 2014).

A crescente importância do setor de serviços no contexto econômico mundial e a necessidade de conquistar clientes para garantir a sobrevivência no mercado demandam a identificação dos critérios segundo os quais os clientes avaliam a excelência do serviço. Dessa forma, o prestador de serviço pode priorizar seus esforços para atender aqueles atributos considerados mais importantes pelos clientes (TINOCO; RIBEIRO, 2008). Albrecht (1992) define qualidade de serviço como a capacidade de uma experiência ou qualquer outro fator que venha satisfazer uma necessidade, resolver um problema ou fornecer benefícios a alguém.

O setor de serviços possui características peculiares que o diferem do industrial, pois muitas vezes, uma organização é resultado da fusão de uma empresa prestadora de serviço com outra de bens, como é o caso das empresas do setor de alimentação, que fornecem a refeição, servida no restaurante, como um produto tangível e a prestação de serviços como um serviço intangível que são as sensações percebidas pelos clientes (PINHEIRO et al., 2008).

Segundo Passador et al. (2006), a busca pela praticidade somada à falta de tempo e a distância entre o local de trabalho e a residência fez com que muitas pessoas deixassem de fazer suas refeições em casa e passaram a comer fora. Com o surgimento dessa demanda, houve um aumento do número de estabelecimentos alimentícios. Nesse sentido há uma preocupação por parte do gestor do estabelecimento em oferecer serviços que estejam adequados às necessidades dos clientes, relacionados com a sua expectativa, tais como, a qualidade da alimentação, o conforto, a localização, 
o atendimento, o custo benefício, entre outros. Pois clientes constroem suas expectativas de pré-consumo, examinam o produto/serviço, relacionam suas expectativas e tiram conclusões baseadas no conhecimento adquirido, formando desse modo, sua avaliação de satisfação (OLIVER, 1993).

O objetivo desse trabalho é avaliar a satisfação dos clientes de um restaurante em Santa Maria - RS, por meio do Modelo Logístico de Dois Parâmetros (ML2) da Teoria da Resposta ao Item (TRI), bem como criar uma escala para medir o nível de satisfação.

\section{REFERENCIAL TEÓRICO}

O termo "satisfação" pode ser definido como "o estado de ser adequadamente recompensado em uma situação de aquisição pelos sacrifícios realizados" (HOWARD; SHETH, 1969). A satisfação também pode ser definida como "o estado psicológico decorrente de um estado emocional aplicado em meio de uma expectativa em virtude de uma aquisição que vem a compor com os sentimentos do consumidor" (OLIVER, 1981) ou ainda "uma completa reação ao ato de consumir" (OLIVER, 1993). De acordo com Kotler (1994), satisfação é o nível de sentimento de uma pessoa resultante da comparação do desempenho ou resultado de um produto (ou serviço) em relação a suas expectativas.

Em restaurantes, a satisfação é percebida pelos serviços prestados, pois resulta de um conjunto de atributos, como a qualidade da comida, a variedade do cardápio, o ambiente físico interno e externo, o tempo de espera, entre outros, pois são esses atributos que compõem a satisfação do cliente, o que determinará se o cliente retornará ou não ao restaurante (DUBE, 1994). Para as empresas, o importante é ter um cliente satisfeito com os seus serviços, de modo que o cliente retorne novamente e até mesmo faça propaganda positiva dos serviços recebidos (BARLOW; MOLLER, 1996). Quando um cliente fica satisfeito com o ambiente isso influencia positivamente, demonstrando assim uma satisfação geral dos clientes para com o restaurante (OLIVER, 1993). Bortolotti et al. (2012) destaca que a satisfação de um consumidor é uma questão de sobrevivência para qualquer organização e que a busca contínua da 
satisfação dos clientes é um dos objetivos mais importantes de um atendimento de boa qualidade.

Por mais que se tenha um foco, um objetivo específico, a avaliação do nível de satisfação das pessoas em relação a um produto ou serviço não é uma tarefa fácil. Os dados sobre os níveis de satisfação dos clientes formam uma das maiores prioridades na administração nas empresas que se comprometem com a qualidade dos seus serviços e produtos, por meio das informações obtidas junto aos seus clientes ou consumidores. Quanto maior o grau de satisfação, maior a lucratividade de uma empresa, muitas vezes chega a ser acima da média. (ROSSI; SLONGO, 1998).

Algumas empresas do ramo de atividades gastronômicas, além de servir alimentos de boa qualidade, devem também ocasionar uma completa experiência sensorial, impecável apresentação do prato, facilidade de manuseio dos talheres, ambiente decorado, iluminação que faça com que o ambiente se torne agradável (SCHMITT, 2000). Outro aspecto observado é que clientes de restaurantes possuem uma tendência de preferir locais onde possam fazer sua alimentação com calma, em baixos níveis de ativação, onde possam relaxar no momento do consumo (WAKEFIELD et al, 1994). Para o cliente é importante ter um local agradável para que se sinta confortável com a qualidade dos serviços, com o ambiente e com a alimentação (BARLOW; MOLLER, 1996).

Clientes de serviços de restaurantes fazem uso de três características para avaliar a qualidade do serviço. A primeira, funcional, referente à qualidade da comida servida, diversidade do cardápio, bebidas, entre outros. A segunda, a mecânica, relacionada ao ambiente, decoração, instalações físicas, layout e iluminação. A terceira, o aspecto humano, caracterizado pelo atendimento, comportamento, aparência dos empregados (WALL; BERRY, 2007).

Para captar a satisfação do cliente, a empresa precisa investir em uma pesquisa de satisfação. Segundo Rossi e Slongo (1998), a pesquisa de satisfação permite criar padrões adequados de atendimento aos clientes, aumentar o índice de retenção dos mesmos, maximizar a sua satisfação e anular o índice de insatisfação, sendo a maneira mais eficiente de se avaliar o desempenho de uma organização. Conforme Bortolotti et 
al. (2012), a pesquisa de satisfação proporciona uma percepção positiva dos consumidores quanto à empresa; relações de fidelidade com os consumidores; dados precisos e atualizados quanto às necessidades dos consumidores; e confiança desenvolvida em função de maior aproximação com o consumidor. Isso faz com que os recursos destinados a aumentar a satisfação do consumidor sejam considerados como investimentos e não como despesas.

Para avaliar a satisfação de clientes existem várias metodologias, dentre as quais, destaca-se a Teoria da Resposta ao Item (TRI). A TRI é uma metodologia que utiliza modelos matemáticos, denominados Modelos de Resposta ao Item (MRI), que permitem estabelecer a relação entre variáveis latentes e suas manifestações (AYALA, 2009), ou seja, é uma forma de representar a relação entre a probabilidade de um indivíduo dar uma resposta a um item e o seu traço latente (ANDRADE; TAVARES; VALLE, 2000), permitindo a criação de uma escala padronizada.

Traços latentes são características do indivíduo que não podem ser medidas diretamente. Por isso, são medidas por meio de um questionário com variáveis secundárias relacionadas com o traço latente, como, proficiência, habilidade, grau de satisfação, importância atribuída, nível de ansiedade, grau de usabilidade, entre outras. (MOREIRA JUNIOR, 2014a).

A TRI foi desenvolvida, principalmente, para suprir limitações da tradicional Teoria Clássica dos Testes (TCT), também chamada de Teoria Clássica de Medidas (TCM), onde o traço latente é mensurado por meio de um somatório ponderado, ou não, dos pontos atribuídos a cada questão do questionário. A TRI apresenta algumas vantagens em relação à TCT, dentre elas destacam-se:

(1) a TRI fornece informações mais precisas do desempenho dos respondentes, tanto em relação à estimação dos parâmetros quanto ao seu erro padrão, já que o traço latente do indivíduo não depende da dificuldade das questões que compõem o questionário, diferente da TCT, onde o escore do indivíduo depende essencialmente do conjunto de itens que compõe o questionário (ANDRADE; TAVARES; VALLE, 2000; EMBRETSON; REISE, 2000; VENDRAMINI; SILVA; CANALE, 2004); 
(2) a TRI permite, sob certas condições, a comparação através do escore entre os indivíduos que respondem questionários com itens diferentes para medir o mesmo traço latente, uma vez que os itens e os indivíduos são colocados numa mesma escala, a qual é interpretável (ANDRADE; TAVARES; VALLE, 2000; EMBRETSON; REISE, 2000);

(3) na TRI, uma vez estimado o traço latente do indivíduo, é possível verificar qual a probabilidade de dar certa resposta a um determinado item, caso ele não o tenha respondido (VENDRAMINI; SILVA; CANALE, 2004).

A TRI surgiu como uma forma de considerar cada item particularmente e não apenas os escores totais, como é feito na TCT, de modo que a TRI não entra em conflito com os princípios da TCT, mas possibilita uma nova proposta de análise estatística (ARAUJO; ANDRADE; BORTOLOTTI, 2009).

Moreira Junior (2014a) descreve de forma sucinta o procedimento geral para uma análise sob a ótica da TRI. O conjunto de itens deve ser elaborado da mesma forma que na TCT, ou seja, por especialistas no assunto do traço latente analisado, consultando a literatura existente sobre o assunto, abrangendo todo o conteúdo necessário, realizando pré-teste e análise de juízes, quando for conveniente. O conjunto de itens resultantes irá compor o questionário, que é o instrumento de avaliação. Esse deverá ser submetido a uma amostra suficiente de respondentes. As respostas obtidas serão analisadas por meio de um MRI, o qual irá fornecer estimativas para os parâmetros dos itens desse modelo. Os itens serão analisados com base nas estimativas e pela Função de Informação do Item (FII), que mede a qualidade do item para o construto. Se algum item tiver qualidade duvidosa, poderá ser eliminado da análise. Os itens com boa qualidade irão se tornar "itens âncoras", conceito que será utilizado nesse trabalho. Após serem encontrados os itens âncoras, será criada a escala do traço latente, onde os itens serão posicionados. Finalizada a avaliação dos itens, a TRI irá avaliar os respondentes, atribuindo uma "nota", ou seja, um valor para o traço latente para cada respondente, que é a estimativa para o parâmetro dos respondentes. Nesse momento, os respondentes poderão ser posicionados na escala criada, junto com todos os itens, podendo-se, então, fazer a análise e interpretação da escala. 
Existem vários MRI utilizados na TRI, diferentes quanto à sua função e à quantidade de parâmetros, e cada um deles é específico para uma situação. Esses modelos podem ser classificados quanto à sua dimensão (unidimensionais ou multidimensionais), quanto ao tipo de traço latente (cumulativo ou não cumulativo), quanto ao tipo de item (dicotômico ou politômico) e quanto ao número de populações envolvidas (MOREIRA JUNIOR, 2011).

Os modelos unidimensionais são utilizados quando se supõe que o objeto de estudo é composto por um único traço latente ou quando esse pode ser representado por uma única dimensão ou fator. Já os modelos multidimensionais são adequados quando se estuda mais de um traço latente ou quando o traço latente não pode ser representado por uma única dimensão (MOREIRA JUNIOR, 2014a). A dimensionalidade pode ser verificada através de uma Análise Fatorial apropriada para dados categorizados (EMBRETSON; REISE, 2000; ANDRADE; TAVARES; VALLE, 2000).

Uma outra suposição dos modelos unidimensionais é a chamada independência local ou independência condicional, a qual assume que para um dado traço latente, as respostas aos diferentes itens do questionário são independentes, ou seja, assim a probabilidade de responder um item é precisamente determinada pelo nível do traço latente do respondente e não por suas respostas a outros itens do conjunto. Esta suposição é fundamental para o processo de estimação dos parâmetros do modelo. No entanto, a unidimensionalidade implica independência local, assim, é suficiente satisfazer a suposição o de unidimensionalidade (ANDRADE; TAVARES; VALLE, 2000; EMBRETSON; REISE, 2000).

A estimação dos parâmetros dos itens se dá por meio de métodos estatísticos complexos, os quais necessitam de métodos computacionais para serem desenvolvidos. Os principais métodos estatísticos utilizados são: o método da máxima verossimilhança (MV), o método bayesiano da esperança a posteriori (EAP) e o método bayesiano da moda a posteriori (MAP).

A escolha do MRI depende basicamente do tipo de item e representa a probabilidade de resposta a um item em função dos parâmetros do próprio item e do traço latente do respondente (TAVARES; ANDRADE; PEREIRA, 2004). No caso de itens 
dicotômicos, onde não há a possibilidade de acerto casual, há dois modelos que podem ser utilizados, o modelo logístico de um parâmetro (ML1), que considera apenas a dificuldade do item, e o modelo logístico de dois parâmetros (ML2), que considera a dificuldade e a discriminação do item.

\section{MATERIAIS E MÉTODOS}

A coleta dos dados foi realizada por meio de um questionário elaborado pelos autores, baseada na literatura utilizada para essa pesquisa. O pré-teste foi realizado nas dependências do restaurante em uma amostra piloto de 30 respondentes. Após o pré-teste, alguns aspectos do instrumento foram melhorados para melhor compreensão dos respondentes. Alguns questionários foram aplicados diretamente aos clientes nas dependências do restaurante, outros preferiram responder on-line ao mesmo questionário que encontrava-se disponibilizado no site Google Docs (www.google.com/docs). O questionário dividia-se em duas partes, a primeira com abordagens sobre informações do perfil do cliente e a segunda sobre o nível de satisfação do cliente em relação a 28 aspectos referentes ao restaurante. Esses 28 aspectos foram medidos em uma escala likert de cinco pontos, com as seguintes categorias: totalmente insatisfeito (1), insatisfeito (2), razoável (3), satisfeito (4) e totalmente satisfeito (5). Ao final do instrumento, foi disponibilizado um espaço para críticas, elogios e sugestões. A coleta foi realizada no período de agosto de 2013 a agosto de 2014, com uma participação de 665 clientes.

Em uma análise prévia, foi feita uma tentativa de ajuste com o Modelo de Resposta Gradual (MRG) de Samejima (1969). No entanto, os dados politômicos originais revelaram-se multidimensionais na análise fatorial. Dessa forma, nesse estudo optou-se por dicotomizar os dados na tentativa de obter unidimensionalidade. As respostas foram agregadas em duas categorias: satisfeito (1), que abrangeu as categorias satisfeito (4) e totalmente satisfeito (5); e não satisfeito (0), que abrangeu as categorias totalmente insatisfeito (1), insatisfeito (2), razoável (3). A análise fatorial foi realizada com os dados dicotomizados para verificar o pressuposto da unidimensionalidade da TRI (ANDRADE; TAVARES; VALLE, 2000). Foi utilizado o método 
de extração por componentes principais sem rotação, com base na matriz tetratórica, recomendada para dados dicotômicos (REVELLE, 2020).

Para a análise dos dados dicotomizados, foi utilizado um modelo logístico unidimensional. Nesse trabalho, considera-se que todos os itens pesquisados não possuem a mesma discriminação. Dessa forma, será utilizado o modelo logístico de dois parâmetros (ML2), desenvolvido por Birnbaum (1968), que é dado por:

$$
\begin{gathered}
P_{i j}=P\left(U_{i j}=1 / \theta_{j}\right)=\frac{1}{1+e^{-D a_{i}\left(\theta_{j}-b_{i}\right)}}, \\
\text { com } i=1,2, \ldots, l, \text { e } j=1,2, \ldots, n, \text { onde, }
\end{gathered}
$$

I é o quantidade de itens no teste);

$n$ é número total de respondentes;

$a_{i}$ é o parâmetro de discriminação (ou de inclinação) do item $i$;

$b_{i}$ é o parâmetro de dificuldade (ou de posição) do item $i$, medido na mesma escala do traço latente e representa o nível do traço latente necessário para que a probabilidade de uma resposta correta ou concordo ou satisfazer as condições do item seja de 0,5;

$\theta_{j}$ pode representar o traço latente do respondente j;

$U_{i j}$ é uma variável dicotômica que assume os valores 1, quando o respondente j responde corretamente, concorda ou satisfaz as condições do item i, ou 0 caso contrário;

$P\left(U_{i j}=1 \mid \theta_{j}\right)$ é a probabilidade do respondente $j$, com seu traço latente $\theta_{j}$, responder sim, estar satisfeito ou concordar como item $i$ e é chamada de Função de Resposta do Item (FRI);

e é a conhecida constante matemática igual a 2,72 .

$D$ é um fator de escala constante, igual a 1 se os parâmetros dos itens são estimados na métrica da Logística, ou igual a 1,7, se os parâmetros dos itens são estimados na métrica da ogiva Normal, que é a distribuição Normal acumulada, por aproximação (nesse estudo, os parâmetros serão analisados pela métrica da Logística, considerando, portanto, $D=1$ ). 
A Figura 1 apresenta um exemplo de uma $\mathrm{CCl}$ do modelo logístico de 2 parâmetros de um item, cujos parâmetros do item são: $a_{i}=1,6, b_{i}=1$, numa escala $(0,1)$, isto é, média zero e desvio padrão 1.

Figura 1 - Exemplo de CCl do modelo Logístico de 2 parâmetros

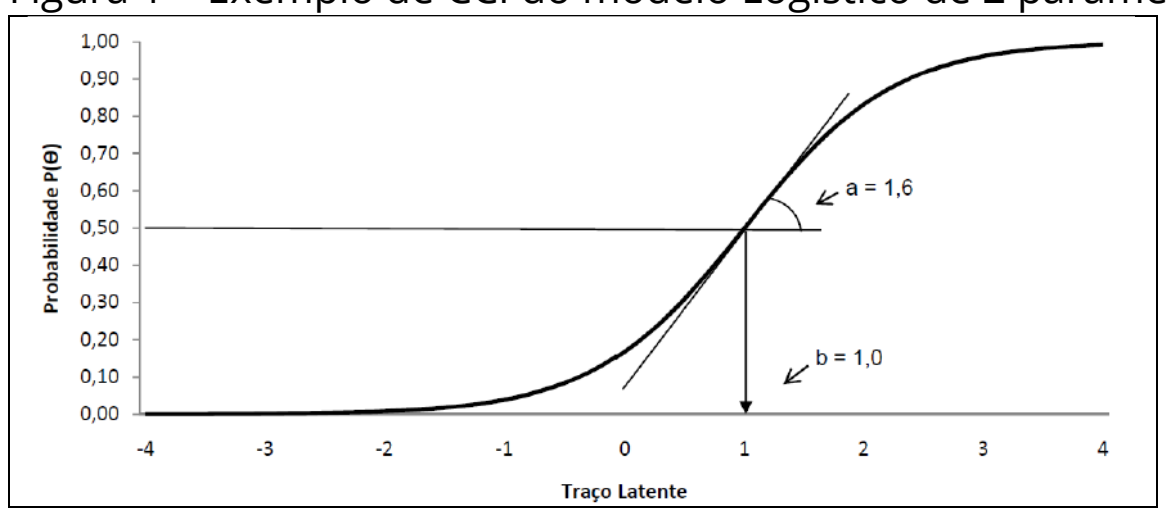

Fonte: Moreira Junior, Tezza e Bornia (2011).

Na Figura 1, pode-se observar que a curva característica do item neste modelo é uma função não linear e, quanto maior a satisfação do indivíduo, maior a probabilidade de responder que está satisfeito com aquele item. Observa-se que, o indivíduo que tem traço latente igual a um, possui 50\% de probabilidade de estar satisfeito com esse item. Indivíduos com traço latente maior que 1 , possuem mais de $50 \%$ de probabilidade de estar satisfeito com esse item, enquanto que indivíduos com traço latente menor que 1, possuem probabilidade menor de $50 \%$ de estar satisfeito com esse item.

O parâmetro $a_{i}$ mede a discriminação do item. Matematicamente, seu valor é proporcional à derivada da tangente da curva no ponto de inflexão, ou seja, no ponto $b_{i}$ (ANDRADE; TAVARES; VALLE, 2000). Valores baixos de $a_{i}$ indicam que 0 item tem pouco poder de discriminação, isto é, a probabilidade de um indivíduo estar satisfeito com ele não difere muito entre os indivíduos satisfeitos ou insatisfeitos. Por outro lado, valores altos de $a_{i}$ indicam que o item tem grande poder de discriminação, dividindo os indivíduos praticamente em dois grupos: os que possuem traço latente abaixo do valor de $b_{i}$ (com baixa probabilidade de concordar ou estar satisfeito com o item), e os que possuem traço latente acima do valor de $b_{i}$ (com alta probabilidade de concordar ou estar satisfeito com o item). Não existe um valor exato de $a_{i}$ para decidir se um item discrimina bem ou não. Os pesquisadores consideram, em geral, que um item com $a_{i}$ maior que 0,7 é aceitável, na métrica logística, porém um valor maior ou igual a 1,0 
indica que o item tem uma boa discriminação. Valores extremamente altos de $a_{i}$ também não são adequados, pois dividiria os indivíduos em dois grupos distintos (os que têm $\theta_{j}$ maior que $b_{i}$ e os que têm $\theta_{j}$ menor que $b_{i}$ ), mas não faria distinção entre os indivíduos dentro dos grupos (MOREIRA JUNIOR, 2014b).

O parâmetro $b_{i}$ é o parâmetro de dificuldade, de posição ou de proficiência do item, que é medido na mesma unidade da escala do traço latente do indivíduo $\left(\theta_{j}\right)$. Quanto maior seu valor, mais difícil é para um indivíduo concordar ou estar satisfeito com o item, e vice-versa. Esse valor de $b_{i}$ é que vai definir a posição do item na escala, por isso ele também é chamado de parâmetro de localização. Teoricamente, $b_{i}$ pode assumir qualquer valor entre $-\infty$ e $+\infty$, no entanto, para valores muito altos ou baixos, o item pode não ser adequado, sendo usual os valores entre -3 e 3 , na escala $(0,1)$, isto é, com média igual a zero e desvio padrão igual a um.

O traço latente do indivíduo $\left(\theta_{j}\right)$ é medido em uma escala arbitrária que varia teoricamente entre $-\infty$ e $+\infty$, diferentemente da TCT, em que a escala geralmente varia entre zero e a quantidade total de questões do questionário. Porém, o importante nessa escala não é a sua magnitude, mas as relações de ordem existentes (ANDRADE; TAVARES; VALLE, 2000). O traço latente, para os modelos acumulativos, é especificado como um tipo de característica que apresenta uma probabilidade maior para indivíduos com $\theta_{j}$ maior, e uma probabilidade menor para indivíduos $\operatorname{com} \theta_{j}$ menor. Ou seja, quanto maior for $\theta_{j}$, maior será a probabilidade do indivíduo $j$ concordar ou estar satisfeito com o item.

Para o ML2, a Função de Informação de um Item (FII) é dada por:

$$
I_{i}(\theta)=a_{i}^{2} P_{i}(\theta) Q_{i}(\theta)
$$

onde:

$$
\begin{aligned}
& P_{i}(\theta)=P\left(U_{i j}=1 \mid \theta_{j}\right) \\
& e \\
& Q_{i}(\theta)=1-P_{i}(\theta) .
\end{aligned}
$$


Observa-se que a informação do item é proporcional ao valor da sua discriminação (parâmetro $a_{i}$ ) e, quanto maior for a discriminação de um item, maior será a informação que ele fornece ao teste ou ao questionário que compõe os itens.

A função de informação total do teste (FIT) é a soma das informações fornecidas por cada item que compõe o mesmo. A curva de informação total é utilizada para avaliar o desempenho dos itens, ou seja, o quão bem um conjunto de itens está avaliando o traço latente. A FIT está relacionada com a precisão necessária para estimar o traço latente, de forma que o erro padrão de medida pode ser estimado como o inverso da raiz quadrada do valor da informação total do teste em cada nível do traço latente. Portanto, quanto maior for a informação, menor será o erro padrão da estimativa do traço latente.

O processo de estimação dos parâmetros dos itens e dos respondentes, no caso do ML2, está implementado em softwares específicos, tais como, BILOG-MG (TOIT, 2003), o XCalibre (GIERL; ACKERMAN, 1996) e em alguns pacotes do software $R(R$ DEVELOPMENT CORE TEAM, 2008) Neste trabalho, os parâmetros do modelo foram estimados por meio do método da Máxima Verossimilhança Marginal, método implementado no pacote irtoys (PARTCHEV, 2013) do software R.

O processo de calibração dos itens, ou seja, a estimação dos parâmetros dos itens, geralmente é feita na escala $(0,1)$, devido à facilidade computacional, ou seja, numa escala com média igual a zero e desvio padrão igual a 1 (MOREIRA JUNIOR, 2014b).

Após essa etapa, é realizada a construção da escala do traço latente, que é colocada na mesma métrica dos itens. Conforme, Fontanive, Elliot e Klein (2007), as escalas de traço latente ordenam o desempenho dos indivíduos do menor para o maior de forma contínua e são cumulativas, isto é, os indivíduos que situam-se em um determinado nível da escala são capazes de demonstrar as habilidades descritas nesse nível e nos níveis anteriores dessa escala

No entanto, existe uma dificuldade, na prática, em compreender os valores negativos e decimais que existem na escala $(0,1)$, na qual muitas vezes os valores das proficiências são interpretados inadequadamente (VALLE, 2001). 
Nesse sentido, é bastante comum fazer uma transformação linear em todos os parâmetros envolvidos antes da construção das escalas, fazendo com que todos os valores fiquem positivos, de forma a representar o traço latente em valores numéricos que tornem mais fácil o entendimento pelas pessoas.

A construção da escala tem o objetivo de encontrar uma interpretação qualitativa dos valores obtidos pela aplicação do modelo da TRI, possibilitando, assim, a interpretação pedagógica dos valores do traço latente. Nesse sentido, surge a ideia dos níveis âncoras e a técnica conhecida como ancoragem (BEATON; ALLEN, 1992).

Andrade, Tavares e Valle (2000) definem níveis âncora como pontos selecionados pelo analista na escala do traço latente para serem interpretados pedagogicamente. Usualmente, os níveis âncoras são estabelecidos em função do desvio padrão em relação à média. Beaton e Allen (1992) definem item âncora da seguinte forma: considere dois níveis âncora consecutivos $Y$ e $Z$, sendo que $Y<Z$. Um determinado item é âncora para o nível $Z$ se e somente se as 3 condições abaixo forem satisfeitas simultaneamente:

$$
\begin{aligned}
& P(U=1 \mid \theta=Z) \geq 0,65, \\
& P(U=1 \mid \theta=Y) \leq 0,50, \\
& P(U=1 \mid \theta=Z)-P(U=1 \mid \theta=Y) \geq 0,30,
\end{aligned}
$$

Isto significa que para um item ser considerado âncora em um determinado nível âncora $Y$ é necessário que seja respondido positivamente por pelo menos $65 \%$ dos respondentes com este nível do traço latente e por uma proporção menor do que 50\% dos respondentes de um nível de traço latente imediatamente inferior X (ANDRADE; TAVARES; VALLE, 2000). E ainda, que a diferença entre a proporção de respondentes destes dois níveis consecutivos deve ser pelo menos de 30\% (Beaton; Allen, 1992). Portanto, um item é considerado âncora quando for típico daquele nível ancora Y e atender as três condições descritas. 
Na prática, às vezes um item não se caracteriza âncora por violar "levemente" uma das três condições necessárias. Nessas situações, pode-se considerar esse item como sendo âncora, se ele for importante ou se existirem poucos itens no instrumento de pesquisa. Outra alternativa é dividir os itens em grupos, segundo a quantidade de condições satisfeitas, ou seja, os itens âncoras que satisfazem as três condições, e os itens "quase" âncoras que satisfazem uma ou duas condições. Depois de identificados os itens âncoras de cada nível âncora, os especialistas no traço latente estudado devem caracterizar os níveis âncoras, segundo o conteúdo abordado no conjunto de itens que compõem cada nível.

Para as análises descritivas foi utilizado o software Excel (MICROSOFT, 2003), para a análise fatorial, utilizou-se o pacote psych (REVELLE, 2020) e, para a análise da TRI, o pacote irtoys (PARTCHEV, 2013), ambos do software $R$ (R DEVELOPMENT CORE TEAM, 2008). As questões não respondidas foram consideradas nas análises da TRI, sendo tratadas como "não apresentadas".

Essa pesquisa faz parte de um projeto acadêmico que foi devidamente avaliado e aprovado pelo Comitê de Ética em Pesquisa da Universidade Federal de Santa Maria (UFSM) registrado sob o número CAE 11183712.4.0000.5346.

\section{RESULTADOS E DISCUSSÃO}

\subsection{Perfil da amostra}

Dentre os 665 clientes abordados, 46,1\% costumam almoçar (a la carte) nesse restaurante, 34,2\% preferem jantar (a la carte) e 19,7\% optam por ir ao buffet que é servido aos domingos. A maior parte dos 640 clientes entrevistados pertence ao sexo masculino (59,3\%), enquanto que apenas $40,7 \%$ são do sexo feminino. Os clientes entrevistados são frequentadores do restaurante há doze anos, em média e a maioria da clientela do restaurante $(58,5 \%)$ é frequentadora há dez anos ou menos. A maior parte dos frequentadores são adultos (32,0\%), com idade entre 36 e 50 anos, mas há também uma boa frequência de adultos jovens (24,1\%), entre 25 e 35 anos, e de adultos maduros $(18,1 \%)$. Cerca de $59 \%$ dos clientes possuem uma renda familiar de até $\mathrm{R} \$$ $6.000,00$, sendo que a maior parcela dos frequentadores possui uma renda familiar 
entre $R \$ 3.000,00$ e $R \$ 6.000,00$ (31,1\%). Há também uma considerável parcela de indivíduos com renda menor $(15,9 \%$ abaixo de $\mathrm{R} \$ 1.900,00)$ e com renda elevada $(25,1 \%$ acima de $\mathrm{R} \$ 12.000,00$ ), indicando que esse restaurante tem uma boa participação das várias classes sociais da cidade, com o predomínio da classe média.

A maior parte dos entrevistados é casado ou com união estável (51\%), 26\% são solteiros, 10\% divorciados, 3\% viúvos e 10\% não declararam. Quanto ao nível escolar, a maior parte (38,2\%), possui curso superior completo, $25,0 \%$ possuem o ensino médio completo e $24,7 \%$ possuem alguma pós-graduação. Isso mostra que o restaurante é frequentado por pessoas com bom nível de escolaridade.

Quase metade dos clientes respondeu que gosta de fazer suas refeições no restaurante devido à qualidade da comida. O atendimento dos funcionários é o segundo principal motivo, sendo apontado por $23,6 \%$ dos clientes. A tradição do restaurante e o ambiente (conforto, ambiente familiar, etc.) também foram motivos citados por mais de $10 \%$ dos clientes.

\subsection{Análise descritiva}

A Tabela 1 apresenta o percentual válido de clientes satisfeitos e não satisfeitos. Observa-se que os maiores índices de satisfação estão relacionados com a infraestrutura do restaurante.

As opções de pagamento foi o item melhor avaliado (93,6\%), seguido pela localização (92,3\%), e pelo tamanho e a estrutura do restaurante (92,2\%). Apenas o item decoração teve mais clientes não satisfeitos (52,3\%) do que satisfeitos. 
Tabela 1 - Análise descritiva dos itens

\begin{tabular}{|c|c|c|}
\hline Item & Satisfeitos & Não Satisfeitos \\
\hline \multicolumn{3}{|l|}{ Ambiente Interno } \\
\hline 1. Tamanho e estrutura do restaurante & $92,2 \%$ & $7,8 \%$ \\
\hline 2. Layout (disposição das mesas e acesso) & $85,1 \%$ & $14,9 \%$ \\
\hline 3. Banheiro & $62,8 \%$ & $37,2 \%$ \\
\hline 4. Limpeza & $72,5 \%$ & $27,5 \%$ \\
\hline 5. Iluminação & $72,4 \%$ & $27,6 \%$ \\
\hline 6. Cheiro & $63,3 \%$ & $36,7 \%$ \\
\hline 7. Decoração & $47,7 \%$ & $52,3 \%$ \\
\hline $\begin{array}{l}\text { 8. Conforto (cadeiras, alturas das mesas, } \\
\text { temperatura) }\end{array}$ & $64,6 \%$ & $35,4 \%$ \\
\hline \multicolumn{3}{|l|}{ Ambiente Externo } \\
\hline 9. Fachada & $80,5 \%$ & $19,5 \%$ \\
\hline 10. Localização & $92,3 \%$ & $7,7 \%$ \\
\hline 11. Acessibilidade & $83,5 \%$ & $16,5 \%$ \\
\hline 12. Sinalização & $73,4 \%$ & $26,6 \%$ \\
\hline 13. Estacionamento & $55,6 \%$ & $44,4 \%$ \\
\hline \multicolumn{3}{|l|}{ Alimentação } \\
\hline 14. Organização do Buffet (distribuição dos pratos) & $84,4 \%$ & $15,6 \%$ \\
\hline 15. Qualidade dos pratos & $86,9 \%$ & $13,1 \%$ \\
\hline 16. Apresentação e identificação dos pratos & $78,7 \%$ & $21,3 \%$ \\
\hline 17. Tempo de espera & $86,1 \%$ & $13,9 \%$ \\
\hline 18. Diversidade de itens do cardápio & $88,8 \%$ & $11,2 \%$ \\
\hline 19. Quantidade das porções & $86,5 \%$ & $13,5 \%$ \\
\hline 20. Opções de pagamento & $93,6 \%$ & $6,4 \%$ \\
\hline 21. Preço cobrado & $81,0 \%$ & $19,0 \%$ \\
\hline 22. Rapidez no pagamento & $88,8 \%$ & $11,2 \%$ \\
\hline \multicolumn{3}{|l|}{ Atendimento Profissional } \\
\hline $\begin{array}{l}\text { 23. Apresentação pessoal dos funcionários } \\
\text { (uniforme, higiene pessoal, postura) }\end{array}$ & $79,3 \%$ & $20,7 \%$ \\
\hline $\begin{array}{l}\text { 24. Domínio e conhecimento sobre os produtos } \\
\text { apresentados no cardápio }\end{array}$ & $85,8 \%$ & $14,2 \%$ \\
\hline 25. Agilidade dos atendentes & $78,1 \%$ & $21,9 \%$ \\
\hline 26. Iniciativa dos atendentes & $75,0 \%$ & $25,0 \%$ \\
\hline 27. Preocupação em atender bem o cliente & $81,5 \%$ & $18,5 \%$ \\
\hline 28. Disponibilidade dos garçons no atendimento & $75,0 \%$ & $25,0 \%$ \\
\hline
\end{tabular}

Fonte: Elaborado pelo autor. 


\subsection{Análise Fatorial}

A Figura 2 apresenta o gráfico Scree Plot da Análise Fatorial, com cinco componentes com autovalor maior que 1. Observa-se a existência de um fator dominante em relação aos demais.

A Tabela 2 apresenta as cargas fatoriais dos 28 itens nas cinco primeiras componentes. Observa-se todos os itens carga fatorial (correlação) positiva com a primeira componente entre 0,42 e 0,84. Apenas o item 9 tem uma carga fatorial um pouco maior em outra componente.

A Tabela 3 apresenta a variância explicada das primeiras componentes. Observase que a primeira componente é dominante pois explica $47 \%$ da variância, quase metade da variância total, enquanto que o segundo explica apenas 13\%. Dessa forma, se satisfaz a exigência da existência de unidimensionalidade, ou de um fator dominante, exigida pelo ML2 da TRI.

Figura 2 - Scree Plot

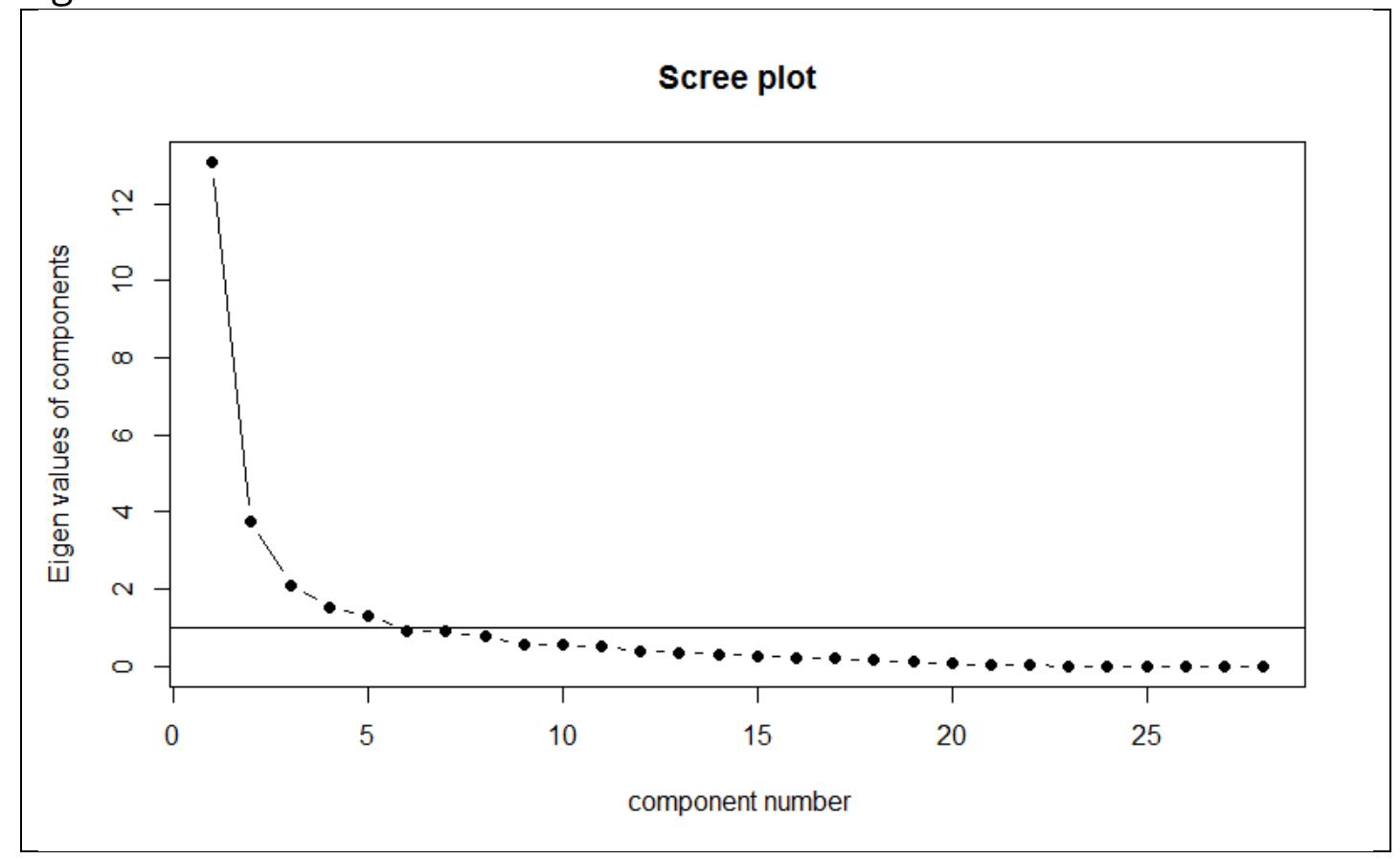


Tabela 2 - Cargas Fatoriais

\begin{tabular}{|c|c|c|c|c|c|}
\hline Item & PC1 & $\mathrm{PC2}$ & PC3 & PC4 & PC5 \\
\hline \multicolumn{6}{|l|}{ Ambiente Interno } \\
\hline 1. Tamanho e estrutura do restaurante & 0,64 & 0,49 & 0,29 & $-0,02$ & $-0,15$ \\
\hline 2. Layout (disposição das mesas e acesso) & 0,55 & 0,53 & 0,33 & 0,18 & $-0,23$ \\
\hline 3. Banheiro & 0,76 & $-0,23$ & 0,01 & 0,26 & $-0,06$ \\
\hline 4. Limpeza & 0,84 & $-0,34$ & 0,02 & 0,27 & $-0,09$ \\
\hline 5. Iluminação & 0,81 & $-0,36$ & 0,10 & 0,22 & 0,01 \\
\hline 6. Cheiro & 0,75 & $-0,35$ & 0,06 & 0,29 & $-0,04$ \\
\hline 7. Decoração & 0,70 & $-0,25$ & 0,34 & 0,32 & $-0,15$ \\
\hline 8. Conforto (cadeiras, alturas das mesas, temperatura) & 0,64 & $-0,06$ & 0,30 & 0,17 & $-0,40$ \\
\hline \multicolumn{6}{|l|}{ Ambiente Externo } \\
\hline 9. Fachada & 0,42 & 0,58 & 0,51 & 0,13 & 0,00 \\
\hline 10. Localizaç & 0,62 & 0,48 & 0,29 & $-0,27$ & 0,15 \\
\hline 11. Acessibilidade & 0,64 & $-0,11$ & 0,47 & $-0,20$ & 0,09 \\
\hline 12. Sinalização & 0,51 & 0,19 & 0,40 & 0,06 & 0,62 \\
\hline 13. Estacionamento & 0,50 & $-0,11$ & 0,22 & 0,06 & 0,54 \\
\hline \multicolumn{6}{|l|}{ Alimentação } \\
\hline 14. Organização do Buffet & 0,51 & 0,13 & $-0,46$ & 0,35 & $-0,01$ \\
\hline 15. Qualidade dos pratos & 0,73 & 0,12 & $-0,43$ & 0,28 & 0,03 \\
\hline 16. Apresentação e identificação dos pratos & 0,68 & 0,09 & $-0,31$ & 0,25 & 0,20 \\
\hline 17. Tempo de espera & 0,57 & 0,48 & $-0,35$ & $-0,12$ & 0,21 \\
\hline 18. Diversidade de itens do cardápio & 0,64 & 0,44 & $-0,17$ & 0,06 & $-0,06$ \\
\hline 19. Quantidade das porções & 0,56 & 0,50 & $-0,28$ & $-0,10$ & 0,15 \\
\hline 20. Opções de pagamento & 0,74 & 0,48 & $-0,17$ & $-0,19$ & $-0,11$ \\
\hline 21. Preço cobrado & 0,71 & 0,24 & $-0,17$ & $-0,31$ & $-0,11$ \\
\hline 22. Rapidez no pagamento & 0,64 & 0,46 & $-0,27$ & 0,03 & $-0,13$ \\
\hline \multicolumn{6}{|l|}{ Atendimento Profissional } \\
\hline $\begin{array}{l}\text { 23. Apresentação pessoal dos funcionários } \\
\text { (uniforme, higiene pessoal, postura) }\end{array}$ & 0,80 & $-0,43$ & $-0,11$ & $-0,21$ & $-0,01$ \\
\hline $\begin{array}{l}\text { 24. Domínio e conhecimento sobre os } \\
\text { produtos apresentados no cardápio }\end{array}$ & 0,71 & $-0,13$ & 0,12 & $-0,54$ & $-0,34$ \\
\hline 25. Agilidade dos atendentes & 0,83 & $-0,40$ & $-0,11$ & $-0,15$ & 0,13 \\
\hline 26. Iniciativa dos atendentes & 0,78 & $-0,41$ & $-0,01$ & $-0,26$ & 0,14 \\
\hline 27. Preocupação em atender bem o cliente & 0,84 & $-0,34$ & $-0,12$ & $-0,16$ & $-0,08$ \\
\hline 28. Disponibilidade dos garçons no atendimento & 0,77 & $-0,50$ & $-0,13$ & $-0,23$ & 0,05 \\
\hline
\end{tabular}

Tabela 3 - Variância explicada das primeiras componentes

\begin{tabular}{lccccc}
\hline & PC1 & PC2 & PC3 & PC4 & PC5 \\
\hline Proporção da variância explicada & 0,47 & 0,13 & 0,08 & 0,05 & 0,05 \\
Variância explicada acumulada & 0,47 & 0,60 & 0,68 & 0,73 & 0,78 \\
\hline
\end{tabular}

\subsection{Análise por meio da TRI}

A Tabela 4 apresenta o valor estimado dos parâmetros de discriminação (a) e de dificuldade (b), assim como os seus respectivos Erros Padrões (EP). As CCl's dos itens 
são apresentadas no gráfico (a) da Figura 3, ao lado das Fll's apresentadas no gráfico (b) da Figura 3.

O menor valor de discriminação $(0,69)$ refere-se ao item "fachada", o que significa que esse item é o que menos discrimina entre os clientes satisfeitos e insatisfeitos. Apesar desse item ter a tido a maior carga fatorial relacionada com a segunda componente, ele não pode ser desprezado, pois segundo Baker (2001), itens com valores entre 0,65 e 1,34 possuem discriminação moderada. Clientes com nível de satisfação -1, têm 0,70 de probabilidade de estarem satisfeitos com esse item, clientes com nível de satisfação 0 têm 0,82 de probabilidade e clientes com satisfação 1 têm 0,90 de probabilidade. As diferenças entre essas probabilidades não é muito grande, pois o item não tem muito poder de discriminação.

Por outro lado, há vários itens com alta discriminação, principalmente relacionados com o atendimento profissional. Esses itens possuem um erro padrão maior que os demais, no entanto, isso é esperado, já que quando maior a discriminação de um item, maior o seu erro padrão, em geral. Apesar do erro padrão maior, esses itens possuem elevada carga fatorial com a primeira componente (Tabela 2), portanto, não podem ser desprezados. O item com a mais alta discriminação $(6,17)$ foi a "agilidade dos atendentes". Clientes com nível de satisfação -1, têm 0,18 de probabilidade de estarem satisfeitos com esse item, mas clientes com nível de satisfação 0 têm 0,99 de probabilidade. A diferença entre essas probabilidades é muito grande, pois o item não forte poder de discriminação. Consequentemente, esse item é o que representa a curva mais elevada no gráfico (b) da Figura 3.

O nível de dificuldade dos itens variou entre $-2,42$ e 0,06. Apenas o item "decoração" obteve um valor de dificuldade positivo, o que corrobora com o fato que esse item foi o único que teve mais de $50 \%$ de não satisfeitos $(52,3 \%)$, conforme a Tabela 1. Clientes com nível de satisfação -1, têm 0,11 de probabilidade de estarem satisfeitos com esse item, clientes com nível de satisfação 0 têm 0,47 de probabilidade e clientes com satisfação 1 têm 0,86 de probabilidade. O item "localização" obteve o menor valor de dificuldade $(-2,42)$. Clientes com nível de satisfação -1 , têm 0,86 de probabilidade de estarem satisfeitos com esse item, clientes com nível de satisfação 0 
têm 0,95 de probabilidade e clientes com satisfação 1 têm 0,99 de probabilidade. Observa-se essas probabilidades altas justamente porque o item tem dificuldade baixa. Observa-se, assim que todos os valores dos parâmetros tanto de discriminação quanto de dificuldade são adequados, o que mostra que todos os itens tiveram um bom ajuste com o ML2.

Tabela 4 - Parâmetros de Discriminação (a) e de Dificuldade (b) dos itens e seus Erros Padrões (EP)

\begin{tabular}{|c|c|c|c|c|}
\hline \multirow[t]{2}{*}{ Item } & \multicolumn{2}{|c|}{ Discriminação } & \multicolumn{2}{|c|}{ Dificuldade } \\
\hline & a & $E P(a)$ & $\mathrm{b}$ & $E P(b)$ \\
\hline \multicolumn{5}{|l|}{ Ambiente Interno } \\
\hline 1. Tamanho e estrutura do restaurante & 1,30 & 0,20 & $-2,36$ & 0,28 \\
\hline 2. Layout (disposição das mesas e acesso) & 1,04 & 0,16 & $-1,99$ & 0,25 \\
\hline 3. Banheiro & 2,17 & 0,23 & $-0,39$ & 0,06 \\
\hline 4. Limpeza & 4,31 & 0,52 & $-0,63$ & 0,05 \\
\hline 5. Iluminação & 3,25 & 0,37 & $-0,66$ & 0,06 \\
\hline 6. Cheiro & 2,41 & 0,25 & $-0,42$ & 0,06 \\
\hline 7. Decoração & 1,94 & 0,20 & 0,06 & 0,07 \\
\hline 8. Conforto (cadeiras, alturas das mesas, temperatura) & 1,49 & 0,16 & $-0,56$ & 0,08 \\
\hline \multicolumn{5}{|l|}{ Ambiente Externo } \\
\hline 9. Fachada & 0,69 & 0,12 & $-2,22$ & 0,37 \\
\hline 10. Localização & 1,26 & 0,20 & $-2,42$ & 0,30 \\
\hline 11. Acessibilidade & 1,50 & 0,19 & $-1,27$ & 0,13 \\
\hline 12. Sinalização & 0,95 & 0,13 & $-1,25$ & 0,17 \\
\hline 13. Estacionamento & 0,97 & 0,12 & $-0,27$ & 0,10 \\
\hline \multicolumn{5}{|l|}{ Alimentação } \\
\hline 14. Organização do Buffet (distribuição dos pratos) & 0,77 & 0,14 & $-0,90$ & 0,18 \\
\hline 15. Qualidade dos pratos & 1,67 & 0,22 & $-1,47$ & 0,14 \\
\hline 16. Apresentação e identificação dos pratos & 1,59 & 0,19 & $-1,07$ & 0,11 \\
\hline 17. Tempo de espera & 1,12 & 0,16 & $-1,83$ & 0,22 \\
\hline 18. Diversidade de itens do cardápio & 1,28 & 0,18 & $-1,84$ & 0,21 \\
\hline 19. Quantidade das porções & 1,00 & 0,15 & $-1,98$ & 0,26 \\
\hline 20. Opções de pagamento & 1,70 & 0,25 & $-2,01$ & 0,21 \\
\hline 21. Preço cobrado & 1,49 & 0,19 & $-1,23$ & 0,13 \\
\hline 22. Rapidez no pagamento & 1,29 & 0,19 & $-1,92$ & 0,22 \\
\hline \multicolumn{5}{|l|}{ Atendimento Profissional } \\
\hline $\begin{array}{l}\text { 23. Apresentação pessoal dos funcionários } \\
\text { (uniforme, higiene pessoal, postura) }\end{array}$ & 5,63 & 0,78 & $-0,79$ & 0,05 \\
\hline 24. Domínio e conhecimento sobre os produtos & 238 & 031 & -124 & 010 \\
\hline 25. Agilidade dos atendentes & 6,17 & 0,89 & $-0,75$ & 0,05 \\
\hline 26. Iniciativa dos atendentes & 4,35 & 0,55 & $-0,70$ & 0,06 \\
\hline 27. Preocupação em atender bem o cliente & 4,88 & 0,66 & $-0,86$ & 0,06 \\
\hline 28. Disponibilidade dos garçons no atendimento & 3,91 & 0,48 & $-0,68$ & 0,06 \\
\hline
\end{tabular}

Fonte: Elaborado pelos autores. 
Figura 3 - CCl's (a) e Fll's (b)

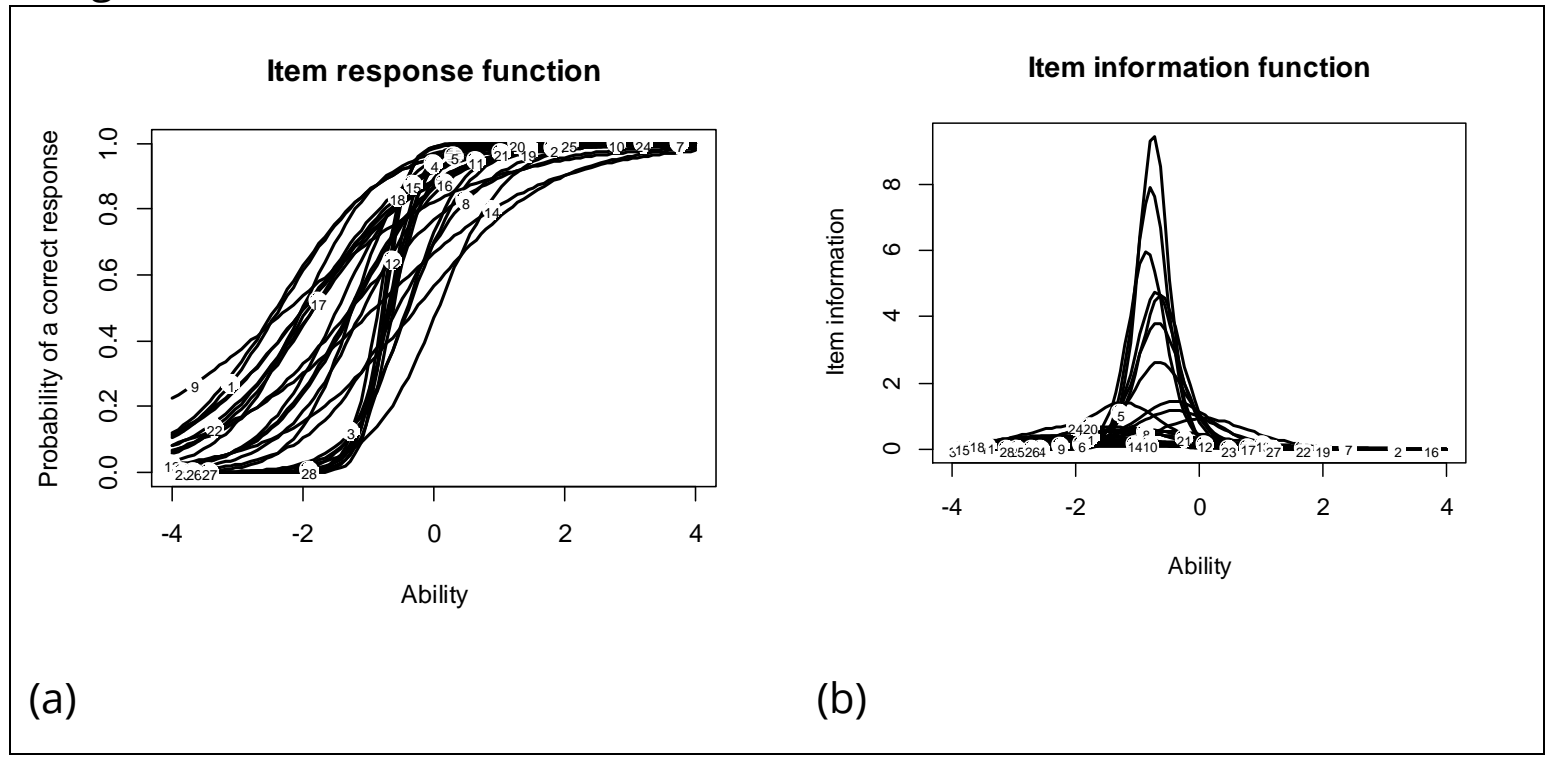

A Figura 4 apresenta a FIT (a) e o erro padrão (b) do traço latente. Por meio da FIT, observa-se que a maior parte da informação concentra-se entre -2 e 0, região onde o erro padrão é menor, com alta concentração de informação um pouco acima do valor -1. Isso mostra que o questionário é mais adequado para medir clientes que têm nível de satisfação entre -2 e 0, abaixo da média da escala. Ou seja, faltam questões para avaliar os clientes satisfeitos (com nível de satisfação acima de zero) de forma mais adequada. O alto pico de informação se justifica pelo fato que há muitos itens com bastante informação um pouco acima de -1 (gráfico $b$ da Figura 3).

Figura 4 - FIT (a) e erro padrão do traço latente (b)

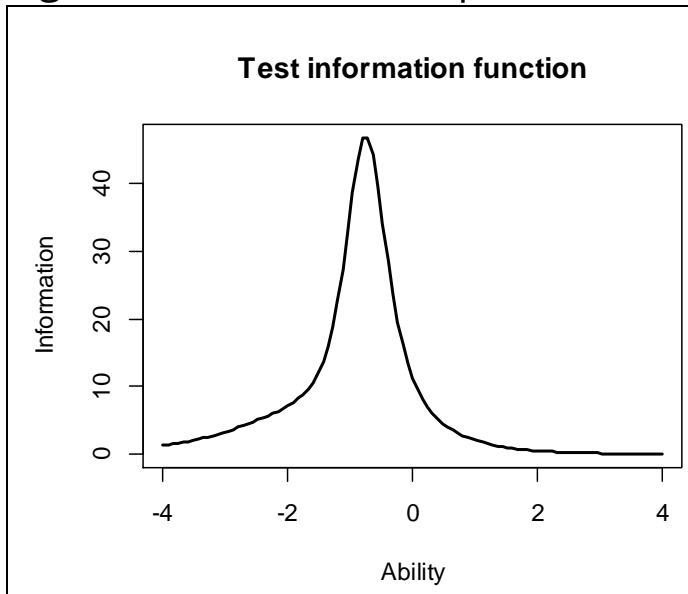

(a)

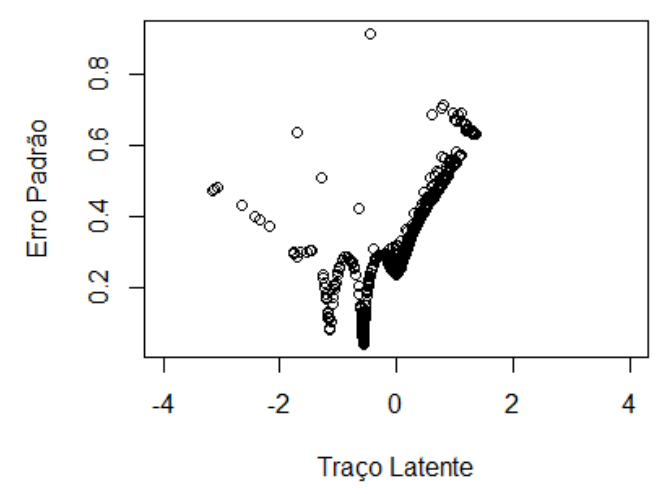

(b) 
A Figura 5 apresenta o Histgrama (a) e o boxplot (b) do traço latente, estimado pelo método EAP. Observa-se que os dados estão praticamente concentrados entre -2 e 2, com alguns poucos dispersos abaixo de -2. O construto conseguiu estimar melhor o traço latente de clientes entre -2 e 0 (mais insatisfeitos), pois o erro padrão é menor (Figura 4-b). Já os clientes entre 0 e 2 (mais satisfeitos) possuem uma estimativa de traço latente com um erro padrão maior. Ou seja, como já foi mencionado, faltam questões para avaliar os clientes satisfeitos (com nível de satisfação acima de zero) de forma mais adequada.

Figura 5 - Histograma (a) e boxplot (b) do traço latente

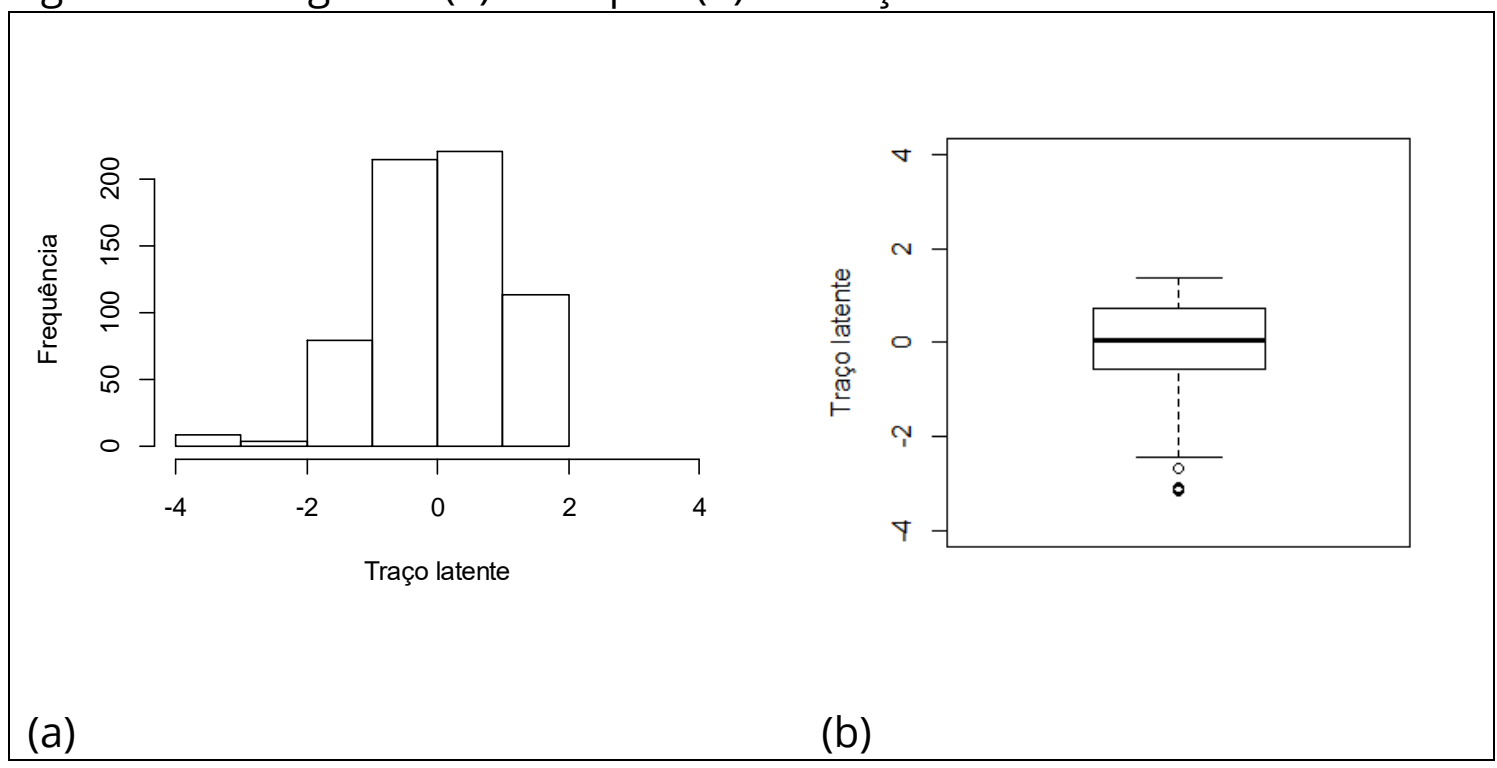

\subsection{Criação da Escala}

Para a caracterização da escala, foram considerados os itens âncoras e os "quase âncoras". Foi possível caracterizar quatro níveis. A Tabela 5 apresenta os níveis âncoras com os seus respectivos itens âncoras e quase âncora.

Tabela 5 - Níveis e Itens Âncoras e Quase Âncoras

\begin{tabular}{lcccc}
\hline Itens\Níveis & -2 & -1 & 0 & 1 \\
\hline Âncoras & - & 15 & $3,4,5,6,8,23,25,26,27,28$ & 7 \\
Quase Âncoras & $1,10,20$ & $2,11,16,17,18,19,21,22,24$ & 14 & - \\
\hline
\end{tabular}

Fonte: Elaborado pelo autor. 
A Interpretação dos níveis âncoras é a seguinte:

Nível -3 ou abaixo: Clientes que estão nesse nível não estão satisfeitos com nenhum item avaliado.

Nível -2: Clientes que estão nesse nível estão satisfeitos apenas com o tamanho e a estrutura do restaurante, com a localização e com as opções de pagamento. $\mathrm{Na}$ pesquisa realizada, $12,5 \%$ dos clientes estavam nesse nível.

Nível -1: Clientes que estão nesse nível estão satisfeitos com todos os itens descritos nos níveis anteriores e com o layout (disposição das mesas e acesso), com a acessibilidade, com a qualidade, apresentação e identificação dos pratos, com o tempo de espera, com a diversidade do cardápio, com a quantidade das porções, com o preço cobrado, com a rapidez no pagamento e com o domínio e conhecimento dos garçons sobre os produtos apresentados no cardápio. Na pesquisa realizada, 33,4\% dos clientes estavam nesse nível.

Nível 0: Clientes que estão nesse nível estão satisfeitos com todos os itens descritos nos níveis anteriores e com os banheiros, a limpeza, a iluminação, o cheiro, com o conforto (cadeiras, alturas das mesas, temperatura), com a organização do buffet (distribuição dos pratos), com a apresentação pessoal dos funcionários (uniforme, higiene pessoal, postura), com a agilidade e a iniciativa dos atendentes, com a preocupação por parte dos funcionários em atender bem o cliente e com a disponibilidade dos garçons no atendimento. Na pesquisa realizada, $34,4 \%$ dos clientes estavam nesse nível.

Nível 1 ou acima: Clientes que estão nesse nível estão satisfeitos com todos os itens descritos nos níveis anteriores e também com a decoração. Na pesquisa realizada, $17,7 \%$ dos clientes estavam nesse nível.

Uma pequena parcela de 2\% de clientes da amostra está abaixo do nível -2. Esses clientes não estão satisfeitos com nenhum item avaliado na pesquisa. 


\section{CONCLUSÕES}

O objetivo desse trabalho foi criar uma escala para avaliar o nível de satisfação dos clientes de um restaurante em Santa Maria - RS, por meio do Modelo Logístico de Dois Parâmetros (ML2) da Teoria da Resposta ao Item (TRI).

Foi aplicado um questionário composto por três partes, a primeira com abordagens sobre informações do perfil do cliente, a segunda sobre o nível de satisfação do cliente em relação a 28 aspectos relacionados ao restaurante, e a terceira parte sobre a importância atribuída pelos clientes a esses 28 aspectos. Esse trabalho delimitou-se a apresentar apenas os resultados relacionados com o nível de satisfação dos clientes. Os clientes foram convidados a avaliar, o seu nível de satisfação, para cada um dos 28 itens, utilizando uma escala likert. Para fins de utilização do ML2, as respostas foram dicotomizadas em duas categorias: satisfeito e não satisfeito. Participaram da pesquisa 640 clientes. Os participantes eram, na sua maioria do sexo masculino, casada ou com união estável, com idade entre 25 e 50 anos, com curso superior completo e freqüentadora há dez anos ou menos.

O resultado da análise fatorial mostrou que existe um fator dominante, satisfazendo a suposição de unidimensionalidade dos dados. O resultado da modelagem por meio do ML2 da TRI mostrou que todos os itens tiveram um bom ajuste, o que revela que a análise é adequada. A FIT mostrou que o questionário é mais adequado para medir clientes que têm nível de satisfação entre - 2 e 0 , abaixo da média da escala. Ou seja, faltam questões para avaliar os clientes satisfeitos de forma mais adequada. O traço latente estimado dos 640 clientes apresenta-se praticamente concentrado entre -2 e 2 . Foi criada uma escala interpretável para a satisfação dos clientes composta por 12 itens âncoras e 13 itens quase âncoras, distribuídos em quatro níveis âncoras.

As escalas elaboradas por meio da TRI são criadas para durarem um bom período de tempo, sem alteração dos parâmetros, onde se beneficiam da propriedade da invariância dos parâmetros dos itens (BAKER, 2001). No entanto, na avaliação da satisfação de clientes, essa propriedade pode não funcionar adequadamente. Por exemplo, os administradores do restaurante poderiam fazer alguma ação para 
melhorar a decoração interna (item mais difícil de obter satisfação). Provavelmente isso mudaria os parâmetros desse item, o que poderia ser verificado numa nova pesquisa de satisfação. Dessa forma, propriedade da invariância dos parâmetros dos itens não seria aplicável, nesse caso.

Pode-se concluir que a TRI é uma ferramenta eficiente para identificar problemas no questionário, como um todo, e nos seus itens específicos, além de criar uma escala onde é possível interpretar o nível de satisfação de cada cliente posicionado nessa escala. Novos clientes podem responder o questionário e serem posicionados na escala, desde que não haja ações que possam mudar as propriedades psicrométricas dos itens. 


\section{REFERÊNCIAS}

ALBRECHT K. Revolução nos serviços. 3. ed. São Paulo: Pioneira, 1992.

ANDRADE DF, TAVARES HR, VALLE RC. Teoria da resposta ao item: conceitos e aplicações. São Paulo: ABE - Associação Brasileira de Estatística, 2000.

ARAUJO EAC, ANDRADE DF, BORTOLOTTI SLV. Teoria da Resposta ao item. Revista da Escola de Enfermagem USP, 43 (Esp):1000-8, 2009.

AYALA RJ. The Theory and Practice of Item Response Theory. The Guilford Press, New York Wiley, 2009.

EMBRETSON S, REISE SP. Item Response Theory for Psychologists. New Jersey: Lawrence Erlbaum Associates, Inc. Publishers, 2000.

BAKER FB. The basis of item response theory parameter estimation techniques. ERIC Clearinghouse on Assessment and Evaluation, 2001.

BARLOW J, MOLLER C. Reclamação de Cliente? Não tem melhor presente: usando o feedback do cliente como uma ferramenta estratégica. São Paulo: Futura, 1996.

BEATON AE, ALLEN NL. Interpreting scales through scale anchoring. Journal of Educational Statistics, 17, 191-204, 1992.

BIRNBAUM A. Some Latent Trait Models and Their Use in Infering an Examinee's Ability. In: LORD FM, NOVICK MR. Statistical Theories of Mental Test Scores. Reading, MA: Addison-Wesley, 1968.

BORTOLOTTI SLV. et al . Consumer satisfaction and item response theory: creating a measurement scale. Gest. Prod., São Carlos, v. 19, n. 2, 2012. Disponível em: http://www.scielo.br/scielo.php?script=sci_arttext\&pid=S0104530X2012000200005\&lng=en\&nrm=iso. Acesso em 06 jul 2014.

DUBE L. et al. Measuring customer satisfaction for strategic management. Cornell Hotel and Restaurant Administration Quarterly, New York: v. 35, n. ํ 1, p.39-48, 1994.

FONTANIVE NS, ELLIOT LG, KLEIN R. Os desafios da apresentação dos resultados da avaliação de sistemas escolares a diferentes públicos. REICE - Revista Electrónica Iberoamericana sobre Calidad, Eficacia y Cambio en Educación, v. 5, n. 2e, 2007.

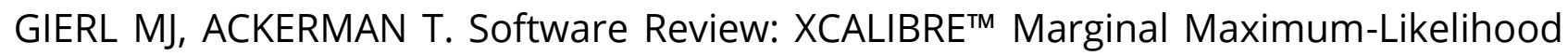
Estimation Program, Windows ${ }^{\mathrm{TM}}$ Version 1. 10. Applied Psychological Measurement, 20, p.303-307, 1996.

HOWARD JA. SHETH JN. The Theory of Buyer Behavior. John Wiley \& Sons, 1969. 
KOTLER P. Administração de Marketing: Analise, Planejamento, Implementação e Controle. 5. ed. São Paulo: Atlas, 1998.

MICROSOFT. Microsoft Excel [computer software]. Redmond, Washington: Microsoft, 2003.

MOREIRA JUNIOR FJ. A utilização da teoria da resposta ao item como ferramenta para a construção de escalas na área de gestão. Revista Gestão Organizacional, v.6, p. 143159, 2014 a.

MOREIRA JUNIOR FJ. Contribuições da Teoria da Resposta ao Item nas Avaliações Educacionais. Ciência e Natura, v. 36, Edição Especial, p. 58-72, 2014b.

MOREIRA JUNIOR FJ. Sistemática para a Implantação de Testes Adaptativos Informatizados baseados na Teoria da Resposta ao Item. 2011. 334 f. Tese (Doutorado em Engenharia de Produção) - Programa de Pós-Graduação em Engenharia de Produção, Centro Tecnológico, Universidade Federal de Santa Catarina, Florianópolis, 2011.

MOREIRA JUNIOR FJ, TEZZA R, BORNIA AC. Estimação da usabilidade de sites ecommerce pelo método da máxima verossimilhança. Ciência e Natura, v. 33, n. 2, p. 23-42, 2011.

OLIVER RL. Measurement and Evaluation of Satisfaction Processes in Retailing Settings. Journal of Retailing, v. 57, n. 3, 1981, p. 25-48.

OLIVER RL. Cognitive, affective, and attribute bases of the satisfaction response. Journal of Consumer Research, Chicago: v. 20, p. 418-430, 1993.

PARTCHEV I. Package irtoys: Simple interface to the estimation and plotting of IRT models, 2013. R package version 0.1.6, 2013.

PASSADOR JL, FILHO DOL, SPANHOL PS, RODRIGUES FS, SABES JJS. A percepção do consumidor de alimentos "fora de casa": um estudo multicaso na cidade de Campo Grande/MS. In: SIMPEP, XIII, Anais..., Bauru, SP, Brasil, 6 a 8 de novembro de 2006.

PINHEIRO FA, LEITE AAM, OLIVEIRA MS. A. Avaliação da Qualidade Percebida em um restaurante temático-típico. Revista Gerenciais, v.7, n.1, p.53-59, São Paulo, 2008.

R DEVELOPMENT CORE TEAM. R: A language and environment for statistical computing. R Foundation for Statistical Computing, Vienna, Austria, 2008.

REVELLE W. Package "psych": Procedures for Psychological, Psychometric, and Personality Research, 2020. R package version 1.9.12.31, 2013. 
ROSSI CAV, SLONGO LA. Pesquisa de Satisfação de clientes: o Estado-da-Arte e Preposição de um Método Brasileiro. Revista de Administração Contemporânea, 1998.

SAMEGIMA F. Estimation of latent ability using a response pattern of graded scores. Psychometric Monograph, 17, 1969.

SCHMITT BH. Marketing experimental: das características e benefícios às experiências. São Paulo: Nobel, 2000.

TAVARES HR, ANDRADE DF, PEREIRA CA. Detection of determinant genes and diagnostic via item response theory. Genetics and Molecular Biology, v. 27, n. 4, p. 679-685, 2004.

TOIT M. IRT from SSI: BILOG-MG, MULTILOG, PARSCALE, TESTFACT. Scientific Software International, 2003.

VALLE RC. A Construção e a Interpretação de Escalas de Conhecimento - Considerações Gerais e uma Visão do que vem sendo feito no SARESP. Estudos em Avaliação Educacional, n. 23, p. 71-92, 2001.

VENDRAMINI CMM, SILVA MC, CANALE M. Análise de Itens de uma Prova de Raciocínio Estatístico. Psicologia em Estudo, Maringá, v. 9, n. 3, p. 487-498, set./dez. 2004.

WAKEFIELD et al. The importance of services capes in leisure service settings. Journal of Services Marketing, Chicago: Vol. 8 No.3, pp.66-76, 2004.

WALL EA, BERRY L. The combined effects of the physical environment and employee behavior on customer perception of restaurant service quality. Cornell Hotel and Restaurant Administration Quarterly, v. 48, n. 1, p.59 - 69, 2007. 